\title{
COMPLICAÇÕES DECORRENTES DO POSICIONAMENTO CIRÚRGICO DE CLIENTES IDOSOS SUBMETIDOS À CIRURGIA DE QUADRILa
}

\author{
Complications arising from surgical positioning in elderly clients submitted to hip surgery. \\ Complicaciones derivadas de la colocación quirúrgica de clientes ancianos sometidos a \\ cirugía de cadera.
}

Andréia Regina Lopes Grigoleto ${ }^{1}$

Maria do Carmo Querido Avelar ${ }^{2}$

Rúbia Aparecida Lacerda ${ }^{3}$

Silvia Helena Frota Mendonça ${ }^{4}$

\section{RESUMO}

Este estudo de revisão sistemática da literatura, segundo as recomendações sugeridas pela Colaboração Cochrane, teve como objetivo buscar nas publicações das bases de dados consultadas as evidências disponíveis sobre complicações decorrentes do posicionamento cirúrgico em idosos submetidos à cirurgia de quadril. Nesta revisão, os estudos identificados e discutidos apresentaram aspectos importantes no desenvolvimento de uma prática efetiva, possibilitando maior compreensão da necessidade de elaboração de pesquisas melhores delineadas que proporcionem validade interna de estudos futuros, para que seus resultados possam ser utilizados na prática clínica.

Palavras-chave: Idoso. Lesões do Quadril. Complicações Intraoperatórias.

\begin{abstract}
The study of this systematic literature review according to the recommendations suggested by Cochrane's Collaboration, had the aim to search in the publications listed in the databases consulted, the available evidences about complications arising from surgical positioning in elderly clients submitted to hip surgery. The studies identified and discussed in this review presented important aspects in the development of an effective practice, enabling greater understanding of the need to elaborate better delineated researches that provide internal validity for future studies, enabling the use of their results in clinical practice.
\end{abstract}

Keywords: Aged. Hip injuries. Intraoperative complications.

\section{Resumen}

El estudio de revisión sistemática de la literatura, siguiendo las recomendaciones de la Colaboración Cochrane, tuvo como objetivo buscar en las publicaciones que figuran en las bases de datos consultadas, las evidencias disponibles para las complicaciones causadas por la colocación quirúrgica en ancianos sometidos a cirugía de cadera. Los estudios identificados y discutidos en esta revisión presentan aspectos importantes en el desarrollo de una práctica eficaz, permitiendo una mayor comprensión de la necesidad de desarrollar estudios mejores delineados que proporcionan validez interna de los futuros estudios, a fin de que sus resultados puedan ser utilizados en la práctica clínica.

Palabras-clave: Anciano. Lesiones de la Cadera. Complicaciones intraoperatorias.

${ }^{1}$ Enfermeira, Mestre em Enfermagem (UnG) Universidade Guarulhos, docente/supervisora de estágio na UNICASTELO - Campus Descalvado, São PauloSP. Brasil. E-mail: andreiarlopes@ig.com.br; ${ }^{2}$ Enfermeira, Doutora em Enfermagem, docente da UnG - Universidade Guarulhos, Guarulhos-SP. Brasil. Email: carmoav@uol.com.br; ${ }^{3}$ Enfermeira, Doutora em Enfermagem, docente titular da Escola de Enfermagem da USP, São Paulo- SP. Brasil. E-mail: rlacerda@usp.br, São Paulo; ${ }^{4}$ Enfermeira, brasileira, Mestre em Enfermagem, Escola de Enfermagem da USP - SP, Enfermeira Chefe dos Serviços Auxiliares de Diagnóstico e Terapêutico Hospital Samaritano-SP, São Paulo-SP. Brasil. E-mail: silviamendonca_604@hotmail.com, 


\section{INTRODUÇÃO}

Com o aumento da expectativa de vida da população idosa, observa-se a ocorrência crescente dos procedimentos anestésico-cirúrgicos em indivíduos desta faixa etária e, consequentemente, maior responsabilidade dos profissionais da área da saúde em se prepararem para um atendimento especializado.

Considerando a necessidade de colocar e manter 0 cliente em variadas posiç̃es na mesa cirúrgica e o intuito de atender, com precisão, as exigências da técnica operatória diante dos resultados desejados, atenção especial deve ser dispensada ao posicionamento do cliente como um ato que exige competência, devendo ser cientificamente preciso e julgado como fator preponderante na realização segura do procedimento cirúrgico. ${ }^{1}$

As informações na área da saúde têm aumentado significativamente, contrastando com a exiguidade do tempo dos profissionais para a busca, análise, comparação e utilização de novos conhecimentos. Esta realidade conduziu ao desenvolvimento de métodos inovadores que auxiliam a utilização de resultados de pesquisas baseadas na prática clínica. ${ }^{2} \mathrm{~A}$ prática clínica baseada em evidências constitui o processo de descoberta, avaliação e uso de achados de investigação com o objetivo de auxiliar nas tomadas de decisões clínicas sobre cuidados em saúde, incorporando princípios, recursos e pessoas. A integração das melhores evidências com a habilidade clínica e a preferência do paciente proporciona uma relação entre diagnóstico e terapêutica, que otimiza o resultado clínico e a qualidade de vida. ${ }^{3}$

As revisões sistemáticas, como metodologia possível de se reproduzir, são investigações cientificas em si mesmas. Como um conjunto de métodos pré-programados sintetizam os resultados de investigações primárias e utilizam estratégias que possibilitam limitar o viés e o erro aleatório. Estas estratégias incluem a pesquisa abrangente de estudos potencialmente relevantes e a utilização de critérios explícitos e reprodutíveis, na seleção desses estudos para revisão. ${ }^{4}$

Na prática clínica, as evidências são importantes para a continuidade do desenvolvimento científico, elevando a qualidade do atendimento aos clientes e avaliando as circunstâncias e os seus desejos, a experiência do profissional e a melhor evidência disponível no momento. ${ }^{5}$

Diante das indagações e questionamentos relacionados à existência de estudos e pesquisas sobre o posicionamento do cliente idoso na mesa de cirurgias, considerando sua maior vulnerabilidade aos riscos que dele decorrem e com a intenção de buscar subsídios básicos para intervenções de enfermagem em sala de operações, estabeleceu-se como objetivo deste estudo realizar uma revisão sistemática da literatura à procura de evidências científicas sobre complicações decorrentes do posicionamento cirúrgico em clientes idosos submetidos à cirurgia de quadril.

\section{MÉTODO}

Neste estudo utilizou-se a revisão sistemática da literatura científica seguindo as recomendações sugeridas pela Colaboração Cochrane, organização internacional sem fins lucrativos responsável pelo preparo, manutenção e por assegurar o acesso às revisões sistemáticas na área da saúde. ${ }^{6}$ Desenvolvido em sete fases, iniciou-se com a formulação da questão abaixo colocada e, para a definição e busca dos descritores, utilizou-se a estratégia $\mathrm{PICO}^{7}$, sendo: P: (paciente ou problema) - idoso, idoso de 80 anos ou mais; I: (intervenção) - artroplastia de quadril, prótese de quadril, articulação do quadril, lesões do quadril, fraturas do quadril, movimento (motion), postura; $C$ : (comparação) componente não utilizado, por não pretender realizar comparações entre procedimentos e 0: ("outcomes" - desfecho) - cuidados intraoperatórios, complicações intraoperatórias. Assim, obteve-se a formulação da seguinte questão: "Há evidências de complicações decorrentes do posicionamento cirúrgico em clientes idosos submetidos a cirurgias de quadril?"

Para a localização e seleção dos estudos foram utilizadas as bases de dados eletrônicas, consideradas pelos centros internacionais de prática baseada em evidências: - PUBMED/ MEDLINE, OVID; CINAHAL; Cochrane e LILACS, por meio dos Descritores em Ciências da Saúde (DECS), nos idiomas inglês, português e espanhol, no período de novembro a dezembro de 2008, seguindo-se, ainda, as especificidades de cada base de dados e com o auxílio das expressões booleanas: AND (intersecção de duas ou mais palavras), $\underline{\mathrm{OR}}$ (localização individual de palavras e ou soma de duas ou mais palavras).

Os critérios de inclusão selecionados para esta revisão sistemática, além das bases de dados já citadas, foram: estudos na íntegra publicados em português; em inglês ou em espanhol; tipo de estudo e ano de publicação; estudos realizados com idosos, ou seja, indivíduos com idade igual ou superior a 65 anos; estudos que abordavam cirurgias de quadril seja por patologias do quadril ou por fraturas de fêmur proximal; estudos que mencionavam complicações decorrentes do posicionamento cirúrgico; estudos realizados no período intraoperatório, em sala de operações, durante o procedimento anestésico-cirúrgico.

A avaliação crítica dos estudos, realizada conforme critérios estabelecidos na revisão, determinou sua inclusão ou a sua exclusão.

Para a coleta de dados dos estudos incluídos na revisão sistemática foi elaborado um instrumento contendo: dados de identificação (título do artigo, título do periódico, autores, país, idioma e ano de publicação); instituição sede do estudo; tipo de revista científica; características metodológicas do estudo (tipo de publicação, objetivo ou questão da investigação, amostra, tratamento de dados, intervenções realizadas, resultados, análise, implicações e nível de evidência); avaliação do rigor metodológico (clareza na identificação da trajetória 
metodológica no texto e identificação de limitações e vieses), tomando como base o instrumento do estudo de Mendonça. ${ }^{8}$

A análise da qualidade das evidências foi realizada de acordo com a seguinte hierarquização: Nível I - metanálise de múltiplos estudos controlados; Nível II - estudo experimental individual; Nível III - estudo quase-experimental como grupo único, não randomizado, controlado, com pré e pós-teste, ou estudos emparelhados tipo caso controle; Nível IV - estudo não experimental como pesquisa descritiva correlacional, pesquisa qualitativa ou estudo de caso; Nível V - relato de casos ou dados obtidos sistematicamente, de qualidade verificável, ou dados de programas de avaliação e Nível VI opinião de autoridades respeitadas (como autores conhecidos nacionalmente) baseada em sua experiência clínica ou a opinião de um comitê de peritos incluindo interpretações das informações relativas à pesquisa e opiniões de órgãos de regulamentação ou legais. ${ }^{9}$

Utilizou-se, ainda, para análise da validade interna de ensaios clínicos randomizados, a Escala de Jadad, pois esta incorpora a abordagem dos mais importantes componentes individuais da qualidade metodológica, incluindo randomização, cegamento, perdas e exclusões. ${ }^{10}$

\section{RESULTADOS}

A distribuição dos estudos, totalizando 662 (100\%), visualizados quantitativamente, conforme a Base de Dados, variou em relação ao número, sobressaindo-se das demais a Base de Dados PUBMED com 401 estudos (60,57\%), seguindose: OVID 112 (16,92\%); COCHRANE 100 (15,11\%); CINAHAL $33(4,98 \%)$ e LILACS 16 (2,42\%).

Dos 662 (100\%) estudos encontrados, utilizando-se as estratégias de busca, em uma primeira reunião de consenso, foram pré-selecionados $47(7,1 \%)$, sendo excluídos 615 (92,9\%), os quais deixaram explícitos pelo título e resumo que não se enquadravam aos critérios propostos. Dos 47 $(7,1 \%)$ estudos selecionados, dois se repetiam entre as diferentes Bases de Dados Eletrônicas, optando-se por excluílos das que obtiveram menor retorno de publicações, mantendoos somente naquela com maior número de retornos, totalizando assim 45 estudos. Estes foram encaminhados à segunda reunião de consenso, que ocorreu após a leitura dos estudos separadamente. Utilizando um instrumento de análise atingiuse um nível de clarificação para inclusão ou não do estudo na presente revisão, resultando assim na exclusão de 43 publicações.

Dos dois estudos incluídos na presente revisão, optouse pela investigação de suas referências, a fim de levantar outros estudos citados que eventualmente não foram encontrados nas Bases de Dados pesquisadas. Na leitura dos títulos das referências, destacaram-se dois novos estudos. Buscou-se então, na Base de Dados PUBMED os dois novos resumos, seguindo-se a localização dos mesmos na íntegra.
Após leitura e análise, os estudos foram excluídos por não atenderem aos critérios estabelecidos.

Os estudos incluídos na revisão sistemática foram nomeados E1 e E7 e encontram-se descritos a seguir: 0 estudo E1, publicado pela revista Anaesthesia, 2007, no Reino Unido, no idioma Inglês, disponível na Base de Dados PUBMED, relata o caso de um idoso submetido à revisão de artroplastia de quadril, em posição lateral, com suporte de apoio abdominal. 0 idoso apresentou hipotensão, sendo necessária intervenção medicamentosa. Foi administrada Efedrina $30 \mathrm{mg}$, com pouca resposta. A seguir, administrou-se Metaraminol e infusão de coloide, com melhora razoável. Somente houve restabelecimento da pressão arterial após a retirada do suporte de apoio abdominal, sem demais complicações. As recomendações foram direcionadas aos anestesiologistas que conduzem cirurgias ortopédicas, os quais devem estar cientes desta potencial complicação. Observouse ausência na descrição do método quanto ao tipo de estudo, razão pela qual foi classificado como relato de caso, designado de "Nível V", na hierarquização dos níveis de evidência."

Do relato de caso $\mathrm{E} 1$ resultou um outro estudo sobre a utilização do suporte de apoio inadequadamente posicionado no abdome, podendo levar à compressão da veia cava e da aorta, propondo-se a utilização de um suporte de apoio colocado na parte superior da crista ilíaca e não contra o abdome. Foi abordada a vantagem em se utilizar este tipo de posicionador em clientes com Índice de Massa Corporal (IMC) acima de 50, tanto na prevenção da compressão abdominal como na estabilização do posicionamento, o que favoreceria a aplicação da anestesia subaracnóidea em posição lateral e a monitorização da pressão venosa central, como altamente desejável em cirurgia ortopédica. ${ }^{12}$

0 estudo E7, publicado pela revista Age Ageing, 1998, no Reino Unido, no idioma Inglês, disponível na Base de Dados PUBMED, incluiu 10 clientes idosos com fratura de fêmur proximal, sendo sete mulheres e três homens, com idade média de 84 anos. 0 intervalo etário constituiu-se de indivíduos entre 67 e 95 anos de idade. 0 procedimento foi realizado em mesa ortopédica, após um tempo de internação entre 2 e 72 horas, com intervalo médio de 12 horas. Foi realizada a avaliação da medida de pressão transcutânea de oxigênio e gás carbônico nas regiões sacra e trocantérica contralateral ao membro operado. Para tanto, utilizaram-se sensores de um monitor radiômetro ligado ao gravador gráfico. Como resultado, observouse uma redução dos níveis transcutâneos de oxigênio abaixo de $20 \mathrm{mmHg}$ em oito dos dez sujeitos do estudo, o que pode produzir comprometimento na viabilidade de tecidos moles. Quanto à pressão transcutânea de gás carbônico, estes foram menos notáveis, excedendo a níveis superiores a $60 \mathrm{mmHg}$ em três dos dez sujeitos da pesquisa. 0 estudo não define o tipo de método utilizado para a realização da pesquisa. No entanto, à análise foi classificado como um ensaio clínico, não randomizado e não cegado. Como conclusão, relata-se que as pressões de gás transcutâneas comprometem os tecidos moles de idosos 


\section{Complicações decorrentes de cirurgia de quadril em idosos}

anestesiados, submetidos a pressões de apoio representativas, o que pode acarretar obstrução nos capilares da pele e isquemia tecidual. As recomendações estavam relacionadas à investigação de novos modelos de superfícies de apoio utilizando-se de medidas objetivas, bem como realização de ensaios clínicos randomizados para a avaliação de uma superfície de apoio eficaz. ${ }^{13}$

Observou-se ausência na descrição do método quanto ao tipo de estudo, sendo necessária a inferência das pesquisadoras por meio da análise da descrição da pesquisa, classificando-a como um ensaio clínico não randomizado, designada, no entanto, como "Nível III", na classificação dos níveis de evidência. Neste caso utilizou-se também a Escala de Jadad, a qual descreve os critérios de validação interna, após avaliação, sendo considerado como de baixa qualidade. $^{10}$

\section{DISCUSSÃO}

Analisando o que foi explicitado acima, pode-se inferir que a responsabilidade do enfermeiro, transdisciplinarmente, com os demais membros da equipe, em sala de operações, está em avaliar previamente o cliente como um todo, bem como observar as condições que decorrem da utilização dos suportes de apoio; o tempo de uso durante o procedimento; $\mathrm{e}$ qualquer alteração que possa estar relacionada ao posicionamento do cliente na mesa de operações.

As intervenções eficazes na prevenção de lesões de pele no período transoperatório estão relacionadas ao alívio de interfaces de pressão durante e imediatamente após a permanência do cliente na mesa cirúrgica, sobre o colchão padrão. 0 uso de dispositivos estáticos à base de polímero de visco elástico seco e colchão a ar apresentam resultados eficazes na prevenção de úlceras de pressão, sendo considerados como alternativas adequadas no alívio das interfaces de pressão. Os colchões ou cober turas de colchão a ar dinâmico, ou sistema micropulsante, poderiam ser considerados os dispositivos mais eficazes na prevenção de lesões de pele no período perioperatório. ${ }^{14}$

Os materiais utilizados para o posicionamento, especialmente acolchoamentos, idealmente deveriam absorver a força de compressão, redistribuir a pressão, prevenir estiramento excessivo e fornecer suporte para uma ótima estabilidade. Estudos sugerem que os dispositivos de posicionamento mantenham a interface pressão capilar normal de $32 \mathrm{mmHg}$ ou menos. ${ }^{15}$

0 valor de $32 \mathrm{~mm} \mathrm{Hg}$ é aceito atualmente como o limiar de pressão externa para além do qual os pequenos vasos entram em colapso, causando trombose, o que resulta em oclusão do fluxo sangüíneo tecidual e privação da quantidade necessária de oxigênio e nutrientes. Ocorre, então, a produção de metabólitos tóxicos a nível celular, levando à acidose tecidual, aumento da permeabilidade capilar, edema, morte celular e formação de ferida por pressão. ${ }^{16}$
Critérios devem ser estabelecidos na escolha dos equipamentos e dispositivos utilizados para posicionar o cliente, os quais incluem: disponibilidade em uma variedade de tamanhos e de formas; durabilidade; capacidade para estar em conformidade com o corpo do cliente; distribuição uniforme, sem qualquer pressão; resistência à umidade e aos microorganismos; radioluminosidade; resistência ao fogo; antialérgico; facilidade de uso; facilidade de limpeza/ desinfecção; facilidade de armazenamento e de manipulação. ${ }^{16}$

Para a aquisição dos dispositivos de proteção, a equipe perioperatória necessita trabalhar de maneira transdisciplinar, objetivando a assistência ao cliente livre de danos relacionados a imperícia, imprudência ou negligência. Quanto ao enfermeiro, é conveniente observar que o método mais eficaz para individualizar a assistência é a Sistematização da Assistência de Enfermagem e, no caso do cliente cirúrgico, a Sistematização da Assistência de Enfermagem Perioperatória (SAEP), utilizada para o levantamento de dados, identificação dos diagnósticos de Enfermagem e a implementação de um plano de cuidados durante o procedimento anestésico-cirúrgico, garantindo a continuidade da assistência. ${ }^{17}$

A presença ou instalação de processos patológicos em idosos podem resultar em alterações que provocam 0 comprometimento da sua estrutura funcional e, consequentemente, no grau de dependência, requerendo, no período mais crítico, aumento da estadia hospitalar, de duração variável para seu tratamento. ${ }^{18}$

As condições preexistentes, especialmente as que afetam os sistemas vasculares, respiratórios, circulatórios, neurológicos e imunitário, bem como os sinais de diminuição da mobilidade ou quaisquer outras condições como problemas de coluna, uso de próteses ou implantes, devem ser consideradas ao criar um plano de atenção para o posicionamento do cliente cirúrgico. Compartilhar as informações com os outros membros da equipe para determinar a melhor maneira de posicionar 0 paciente antes, durante e após o procedimento, utilizando recursos manuais de procedimentos ou outros, deve ser a política do enfermeiro de centro cirúrgico. ${ }^{15}$

Itens elementares deverão ser considerados em estudos futuros desta natureza, incluindo aqueles para os quais se deve atentar pela sua maior pertinência, como: classificação dos idosos por faixa etária; o sexo; o controle do IMC; o registro de doenças comórbidas; o exame físico e a avaliação funcional; os achados considerados fatores de risco como condições da pele; limitações físicas; o modelo da mesa operatória; a posição adotada para cirurgia; os recursos de proteção: dispositivos, acomodadores, posicionadores - tipo, material; o tempo de cirurgia; dados referentes à equipe operatória durante o procedimento cirúrgico; complicações ocorridas pelo posicionamento cirúrgico, se registradas e confirmadas, e outros.

A análise destes dados enfatiza os cuidados com 0 posicionamento do cliente idoso, o qual apresenta seu organismo, 
como um todo, fragilizado pelo passar dos anos. Reforça-se, ainda, a importância de se avaliar cada cliente de forma individualizada, levando em conta as particularidades encontradas durante a coleta de dados e exame físico, o qual deverá incluir as avaliações funcionais espećificas desta faixa etária.

\section{CONCLUSÃO}

Nesta pesquisa não foi possível localizar estudos com força de evidência para afirmar que as complicações identificadas nos trabalhos mencionados foram decorrentes do posicionamento cirúrgico em idosos submetidos à cirurgia de quadril, uma vez que o estudo $E 1$ foi classificado na escala de hierarquização dos níveis de evidências como um Relato de Caso "Nível V", e o estudo E7 foi classificado como ensaio clínico não randomizado, designado como de "Nivel III" na escala de hierarquização dos níveis de evidência, sendo, ainda, avaliado como ensaio clínico de baixa qualidade na Escala de Jadad.

Estudos com o intuito de verificar a eficácia de intervenções específicas de prevenção no período intraoperatório, ou seja, durante o procedimento anestésicocirúrgico de cirurgia de quadril em clientes idosos, seria de grande valia, principalmente se além dos controles das variáveis já citadas fosse utilizado como método o ensaio clínico randomizado, controlado, realizado em instituições com grande fluxo deste tipo de ocorrência, favorecendo, ainda, que o estudo seja unicêntrico e com amostra representativa.

\section{REFERÊNCIAS}

1 - Ricker LE. Posicionamento do paciente cirúrgico. In: Meeker MH, Rothrock JC. Alexander: cuidados de enfermagem ao paciente cirúrgico. $10^{\mathrm{a}}$ ed. Rio de Janeiro(RJ): Guanabara Koogan; 1997. p. 90-105.

2 - Bernardo WM, Nobre MRC, Jatene FB. A prática clínica baseada a em evidências: buscando as evidências em fontes de informação. Rev Assoc Med Bras. 2004; 50(1):104-08.

3 - Sackett DL, Rosenberg WM, Gray JA, Haynes RB, Richardson WS. Evidence based medicine: what it is and what is isn't. British Medical Journal.1996 Jan 13; 312 (7023):71-2.

4 - Cook DJ, Mulrow CD, Haynes RB. Systematic reviews: synthesis of best evidence for clinical decisions. Systematic Review Series. [Internet]. 1997. [cited 2009 Jan 01]; 126(5):376-380. Available from: http:// uww.annals.org/cgi/content/full/126/5/376.

5 - El Dib RP. Como praticar a medicina baseada em evidências. J Vasc Bras. 2007; 6(1):1-4.

6 - Castro AA, Saconato H, Guidugli F, Clark OAC. Curso de revisão sistemática e metanálise. [Internet]. 2002. [citado 2008 out 20]; São Paulo (SP): LEDDIS/UNIFESP. Disponivel em: www.virtual.epm.br/cursos/metanalise.
7 - Santos CMC, Pimenta CAM, Nobre MRC. A estratégia PICO para a construção da pergunta de pesquisa e busca de evidências. Rev Latinoam Enfermagem. 2007; 15(3): 508-11.

8 - Mendonça SHF. Impacto do uso de conectores sem agulha para sistema fechado de infusão na ocorrência de infecção de corrente sanguínea relacionada ao cateter venoso central: evidências de uma revisão sistemática [dissertação]. São Paulo (SP): Escola de Enfermagem, Universidade de São Paulo; 2008. 164p.

9 - Stetler CB, Morsi D, Rucki S, Broughton S, Corrigan B, Fitzgerald J, et al. Utilization-focused integrative reviews in a nursing service. Appl Nurs Rev. 1998; 11(4):195-206.

10 - Jadad AR, Moore RA, Carroll D, Jenkinson C, Reynolds DJ, Gavaghan $D J$, et al. ssessing the quality of reports of randomized clinical trials: is blinding necessary? Control Clin Trials. 1996; 17(1):1-12.

11 - Satisha M, Evans R. Venocaval compression due to abdominal post support and positioning during orthopaedic anaesthesia. Anaesthesia. 2007; 62:1075-081.

12 - Girgis Y, Dunlop DJ. Venocaval compression due to an abdominal post support. Anaesthesia. 2008; 63: 316-27.

13 - Bader DL, White SH. The viability of soft tissues in elderly subjects undergoing hip surgery. Age and Ageing. 1998; 27: 217-21.

14 - Ursi ES, Galvão CM. Prevenção de lesões de pele no perioperatório: revisão integrativa da literatura. Rev Latino-am Enfermagem. 2006; 14(1):124-31.

15 - Millsaps C. Pay attention to patient positioning! Preventing injury during surgery and other procedures is a nursing priority. these pointers will ensure your care is up to snuff. [Internet]. [cited 2009 Jan 20]. Available from: http:/ /rn.modernmedicine.com/rnweb/article/articleDetail.jsp?id=278192

16 - Pfiedler Enterprises'. Basic principles of patient positioning: a continuing education self-study activity. [Internet]. [cited 2009 Feb 22]. Available from: www.pfiedlerenterprises.com.

17-Sociedade Brasileira de Enfermeiros de Centro Cirúrgico: recuperação anestésica e centro de material e esterilização - SOBECC. Práticas recomendadas. $5^{a}$ ed. São Paulo: SOBECC; 2009.

18- BrazE, CiosakSI. 0 tornar-se cuidadora na senescência. Esc Anna Nery Rev Enferm 2009 abr/jun; 13 (2): 372-77.

\section{NOTA}

aArtigo extraído de tese de dissertação de Mestrado Acadêmico em Enfermagem apresentada a Universidade Guarulhos -UnG, Guarulhos, SP, Brasil. 